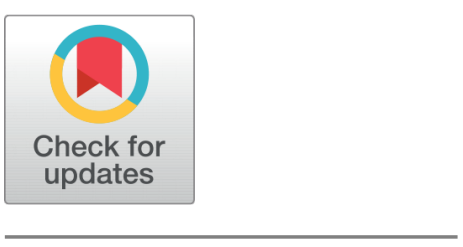

open ACCESS

Received: 25.01.2021

Accepted: 03.05.2021

Published: 19.06.2021

Citation: Sunitha T, Nagi Reddy U, Shobhalatha G (2021) A Note on Full k-Ideals in Ternary Semirings. Indian Journal of Science and Technology 14(21): 1786-1790. https ://doi.org/10.17485/IJST/v14i21.150

* Corresponding author.

nagireddyppr@gmail.com

Funding: None

Competing Interests: None

Copyright: @ 2021 Sunitha et al. This is an open access article distributed under the terms of the Creative Commons Attribution License, which permits unrestricted use, distribution, and reproduction in any medium, provided the original author and source are credited.

Published By Indian Society for Education and Environment (iSee)

ISSN

Print: 0974-6846

Electronic: 0974-5645

\section{A Note on Full k-Ideals in Ternary Semirings}

\author{
T Sunitha ${ }^{1,2}$, U Nagi Reddy ${ }^{*}$, G Shobhalatha ${ }^{4}$ \\ 1 Research Scholar, Department of Mathematics, Rayalaseema University, Kurnool, Andhra \\ Pradesh \\ 2 Lecturer in Govt. Degree College, Nandikotkur, Kurnool, Andhra Pradesh \\ 3 Assistant Professor, Department of Mathematics, Rayalaseema University, Kurnool, Andhra \\ Pradesh \\ 4 Professor, Department of Mathematics, Sri Krishnadevaraya University, Anantapur, Andhra \\ Pradesh
}

\section{Abstract}

Objectives: $\mathrm{k}$ - ideals plays a vital role in ternary semirings. Ternary algebraic systems is a generalization of algebraic structures and it is the most natural way for the further development, deeper understanding of their properties. Methods: We have imposed Integral Multiple Property (IMP) and some other different constrains on a ternary semiring. Findings: In this study, we have described more results on the full k-ideal in the ternary semirings. Finally, we provide the characterization of full k-ideal in ternary semirings and studied their related properties. Applications: The structures of ideals in ternary semirings are widely applicable to computer sciences, dynamical and logical systems, cryptography, graph theory and artificial intelligence.

Keywords: Ternary Semiring; Ideal; k- Ideal; Full k- Ideal; Inverse

\section{Introduction}

The first formal definition of semiring was introduced in the year 1934 by Vandiver ${ }^{(1)}$. Several researches have characterized the many type of ideals on the algebraic structures such as: In 1958, Iséki considered and proved some theorems on quasi-ideals in semirings. However the developments of the theory in semirings have been taking place since 1950. A semiring is basic structure in Mathematics. The semiring theory and semigroup theory influenced on the developments of the semiring theory and its ordering. Nagi Reddy U, Rajani K, and Shobhalatha G have studied the fuzzy bi-ideals in ternary semigroups ${ }^{(2)}$. Ternary rings are introduced with their structures ${ }^{(3)}$. Some properties of ternary semirings are derived with the quasi ideals and Bi ideals ${ }^{(4)}$. $S^{*}$ semirings and $\mathrm{A}^{*}$ semirings, which are studied with the some special structures ${ }^{(5)}$. Certain type of ring congruences on an additive inversive semirings with the help of full k-ideals is studied ${ }^{(6)}$. Sen and Adhikari gave some characterizations of maximal k-ideals of semiring.

Our main purpose of this paper is to introduce the notions of k- ideals and full $\mathrm{k}$ ideals in ternary semirings and study the set of all full k-ideals of an additively inverse ternary semiring in which addition is commutative forms a complete lattice which is 
also modular.

\section{Preliminaries}

Definition 2.1: A Ternary semiring is a nonempty set $S$ together with the binary operation addition and ternary operation multiplication denoted by,$+ \cdot$ respectively, satisfying the following conditions:

1. $(S,+)$ is a commutative semigroup.

2. $(S, \cdot)$ is ternary semigroup.

3. Distributive laws holds, i.e., $a \cdot b(c+d)=a \cdot b . c+a \cdot b . d \quad a(b+c) d=a \cdot b \cdot d+a . c . d$ and $(a+b) \cdot c \cdot d=a \cdot c \cdot d+b \cdot c \cdot d$

Definition 2.2: An element a of a ternary semiring $S$ is said to be additive idempotent element provided a+a=a .

Note: The set of all additive idempotent elements in a ternary semiring $S$ is denoted by $E^{+}(S)$.

That is $E^{+}(S)=\{a \in S / a+a=a\}$.

Definition 2.3: A ternary semiring $S$ is called E-inverse, if for every $a \in S$, there exists $x \in S$ such that $a+x \in E^{+}(S)$.

Note : Let $\mathrm{S}$ be a ternary semi ring, then $E^{+}(S)$ is an ideal of $\mathrm{S}$.

Definition 2.4: A subset I of a ternary semiring $S$ is called a left (resp. a right, lateral) ideal of $S$ if

1. $a+b \in I$ for all $a, b \in I$

2. for any $a \in I$, and $b, c \in S, b c a \in I$ ( resp. $a b c \in I, b a c \in I)$

A subset I is called an ideal if I is left, lateral and right ideal.

Note:

1. If $A, B$ are any two ideals of a ternary semiring $\mathrm{S}$, then $A \cap B$ is an ideal.

2. Let $A, B$ be two ideals of a ternary semiring $\mathrm{S}$, then the sum of $A, B$ denoted by $A+B$ is an ideal of $\mathrm{S}$ where $A+B=\{x=$ $a+b \mid a \in A, b \in B\}$

Definition 2.5: An ideal I of a ternary semiring $S$ is called full if $E^{+}(S) \subseteq I$

Example: In any ternary ring $\mathrm{R}$, the set $E^{+}(R)=\{0\}$, and so every ideal of $\mathrm{R}$ is a full ideal.

Definition 2.6. An ideal I of a ternary semiring $\mathrm{S}$ is called k-ideal or subtractive if for any two elements $a \in I$ and $x \in S$ such that $a+x \in I \quad$, then $x \in I$.

Example. In any ternary ring $\mathrm{R}$, every ideal $\mathrm{I}$ is k-ideal, since for any $a \in I, x \in R$ such that $a+x \in I$ then $a+x+(-a) \in$ $I$ so $x \in I$

Definition 2.7. A k-ideal I of a ternary semiring $S$ is called full k-ideal if the set of all additive idempotents of $S, E^{+}(S)$ is contained in I.

Example 1: In any ternary ring R every ideal $\mathrm{I}$ is a full k-ideal. Since 0 is the only additive idempotent element in $\mathrm{R}$ which belongs to any ideal I of R. So I is full k-ideal.

Example 2: In a distributive lattice $\mathrm{L}$ with more than two elements, a proper ideal $\mathrm{I}$ is $\mathrm{k}$-ideal but not full k-ideal. Let $a \in I, x \in L$ such that $a \vee x \in I$, then $x \leq a \vee x$. But I is an ideal so $x=x \wedge(a \vee x) \in I$. Hence I is k-ideal. Moreover, the set of all additive idempotents of $\mathrm{L}$ is L itself, since $a \vee a=a$ for all $a \in L$. So I is not full k-ideal.

Example 3: In $Z \times Z^{+}=\{(a, b): a, b$ are integers and $b>0\}$ we define $(a, b)+(c, d)=(a+c, l c m(b, d))$ and $(a, b) \cdot(c, d)$. $(e, f)=($ a.c.e, $\operatorname{gcd}(b, d, f))$, then $Z \times Z^{+}$is an additive inversive ternary semiring.

Solution: Let $(a, b),(c, d),(e, f) \in Z \times Z^{+}$

Additive commutative:

$$
(a, b)+(c, d)=(a+c, \operatorname{lcm}(b, d))=(c+a, \operatorname{lcm}(b, d))=(c, a)+(a, b)
$$

Additive associative:

$$
\begin{aligned}
((a, b)+(c, d))+ & (e, f)=((a+c, \operatorname{lcm}(b, d))+(e, f) \\
& =(((a+c)+e, \operatorname{lcm}(\operatorname{lcm}(b, d), f)) \\
& =((a+(c+e), \operatorname{lcm}(b, \operatorname{lcm}(d, f))) \\
& =(a, b)+((c+e), \operatorname{lcm}(d, f)) \\
& =(a, b)+((c, d)+(e, f)) .
\end{aligned}
$$


Multiplicative associative: Similarly as additive associative Distributive:

$$
\begin{aligned}
(a, b) \cdot(c, d)((e, f))+ & (g, h))=(a, b) \cdot(c, d)(e+g, \operatorname{lcm}(f, h)) \\
& =(a \cdot c(e+g), \operatorname{gcd}(b, d, l c m(f, h))) \\
& =(a \cdot c \cdot e+a \cdot c \cdot g, \quad l c m(\operatorname{gcd}(b, d, f), \operatorname{gcd}(b, d, h))) \\
& =(a \cdot c \cdot e, \operatorname{gcd}(b, d, f))+(a \cdot c \cdot e, \operatorname{gcd}(b, d, h)) \\
& =((a, b) \cdot(c, d)(e, f)+(a, b) \cdot(c, d)(g, h))
\end{aligned}
$$

Similarly, $((e, f))+(g, h))(a, b) \cdot(c, d)=((e, f)(a, b) \cdot(c, d)+(g, h)(a, b) \cdot(c, d))$

Additive inverse: For any $(a, b) \in Z \times Z^{+}$there exists a unique $(-a, b) \in Z \times Z^{+}$such that

$$
\begin{aligned}
& (a, b)+(-a, b)+(a, b)=(a+-a+a, \operatorname{lcm}(b, b, b))=(a, b), \\
& (-a, b)+(a, b)+(-a, b)=(-a+a+-a, \operatorname{lcm}(b, b, b))=(-a, b)
\end{aligned}
$$

Moreover, the set $A=\left\{(a, b) \in Z \times Z^{+}-a=0, b \in Z^{+}\right\}$is full k-ideal of $Z \in Z^{+}$.

Since $E^{+}\left(Z \times Z^{+}\right)=\{0\} \times Z^{+} \subseteq A$ and for any $(0, b) \in A,(c, d) \in Z \times Z^{+}$such that $(0, b)+(c, d)=(c, l c m(b, d)) \in A$, then $c=0$, so $(c, d) \in A$.

Definition 2.8: Let $A$ be an ideal of an additive inversive ternary semiring S. We define the k-closure of A, denoted by A by:

$$
\bar{A}=\{a \in S \cdot a+x \in A \quad \text { for some } x \in A\}
$$

Definition 2.9: A lattice $\mathrm{L}$ is called a modular lattice simply modular, if for $a, b, c \in L, a \leq b \quad a \wedge c=b \wedge c \quad a \vee c=$ $b \vee c$ implies $a=b$.

\section{Main Results}

Theorem 3.1. Let A and B be two full k-ideal of a ternary semiring $\mathrm{S}$. then $A \cap B$ is full k-ideal.

Proof. Let A and B be two full k-ideal of S, then $A \cap B$ is an kideal which is full as $E^{+}(S) \subseteq A$ and $E^{+}(S) \subseteq B$

Let $x \in S$ such that $a+x \in A \cap B$ for some $a \in A \cap B$. Then $a+x \in A, a \in A$ and $a+x \in B, a \in B$ which implies that $x \in A$ and $x \in B$.

Hence, $x \in A \cap B$

Therefore, $A \cap B$ is full k-ideal.

Theorem 3.2. Every k-ideal of ternary semiring $S$ is an inversive sub semiring of $S$.

Proof. Clearly that every ideal of $S$ is sub semiring of S. Let $a \in I$, then $a \in S$, so there exist $a^{\prime} \in S$ such that $a=a+a^{\prime}+a=$ $a+\left(a^{\prime}+a\right) \in I$.

But $\mathrm{I}$ is a k-ideal and $a \in I$, so $\quad a^{\prime}+a \in I$. Again $\mathrm{I}$ is a k-ideal and $a \in I$, so $\quad a^{\prime} \in I$.

Hence I is an inversive sub semiring of $S$.

Theorem 3.3. Let A be an ideal of ternary semirin S. Then $A$ is a $k$-ideal of S. Moreover $A \subseteq \bar{A}$.

Proof. Let $a, b \in \bar{A}$, then $a+x, b+y \in A$ for some $x, y \in A$.

Now $(a+b)+(x+y)=(a+x)+(b+y) \in A$.

But $x+y \in A$, so $a+b \in \bar{A}$. Next let $p, r \in S$, then pra $+\operatorname{prx}=\operatorname{pr}(a+x) \in A$.

But prx $\in A$, so, pra $\in \bar{A}$. Similarly, apr $\in A$.

Since $\bar{A}$ is an ideal of $\mathrm{S}$.

To show that $\bar{A}$ is k-ideal.

Let $c, c+d \in \bar{A}$, then there exist $\mathrm{x}$ and $\mathrm{y}$ in A such that $c+x \in A$ and $c+d+y \in A$.

Now $d+(c+x+y)=(c+d+y)+x \in A$ and $c+x+y \in A$.

Hence $d \in \bar{A}$ and so $\bar{A}$ is a k-ideal of S.

Finally, since $a+a \in A$ for all $a \in A$, it follows that $A \subseteq \bar{A}$.

Corollary 3.1 Let A be an ideal of ternary semiring S. Then $\bar{A}=A$ if and only if $\bar{A}$ is a k-ideal.

Proof. Suppose $\bar{A}=A$, then by theorem $3.3 \bar{A}$ is k-ideal, and so A is k-ideal.

Conversely, assume that $\mathrm{A}$ is a k-ideal. Again by theorem $3.3 A \subseteq \bar{A}$. $A=\bar{A}$.

On the other hand, let $a \in \bar{A}$ then $a+x \in A$ for some $x \in A$. But A is a k-ideal and $x \in A$, implies $a \in A$, so $\bar{A} \subseteq A$. Therefore

Corollary 3.2: Let $\mathrm{A}$ and $\mathrm{B}$ be two ideals of a ternary semiring $\mathrm{S}$ such that $A \subseteq B$, Then $\bar{A} \subseteq \bar{B}$. 
Proof. Let $\mathrm{A}$ and $\mathrm{B}$ be two ideals of S such that $A \subseteq B$, let $a \in \bar{A}$, then $a+x \in A$ for some $x \in A$, but $\quad A \subseteq B$, so $a+x \in$ $B$ for some $x \in B$.

Hence $a \in \bar{B}$, Therefore $\bar{A} \subseteq \bar{B}$.

Corollary 3.3: Let A be an ideal of ternary semiring S. Then $\bar{A}$ is the smallest k-ideal containing A.

Proof. Let B be a k-ideal of $\mathrm{S}$ such that $A \subseteq B$, let $x \in \bar{A}$. Then $x+a_{1}=a_{2}$ for some $a_{1}, a_{2} \in A$.

Since $A \subseteq B$ and $B$ is a k-ideal, then $x \in B$.

This implies that $\bar{A} \subseteq B$.

Therefore $\bar{A}$ is the smallest k-ideal containing A.

Theorem 3.4: Let $\mathrm{A}$ and $\mathrm{B}$ be two full k-ideals of ternary semiring $\mathrm{S}$. then $\overline{A+B}$ is a full k-ideal of $\mathrm{S}$ such that $A \subseteq$ $\overline{A+B}$ and $B \subseteq \overline{A+B}$.

Proof. Let $\mathrm{A}$ and $\mathrm{B}$ be two full k-ideals of ternary semiring $\mathrm{S}$. Then $A+B$ is an ideal of $\mathrm{S}$.

Then by theorem $3.3 \overline{A+B}$ is a k-ideal and $A+B \subseteq \overline{A+B}$.

Now $E^{+}(S) \subseteq A$ and $E^{+}(S) \subseteq B$. So for any $e \in E^{+}(S), e=e+e$.

Hence $E^{+}(S) \subseteq A+B \subseteq \overline{A+B}$. Which implies that $\overline{A+B}$ is a full k-ideal.

Finally let $a \in \bar{A}$, Then $a=a+a^{\prime}+a=a+\left(a^{\prime}+a\right) \in A+B \quad$ as $a^{\prime}+a \in E^{+}(S) \subseteq B$

Hence $A \subseteq \overline{A+B}$ and similarly $B \subseteq \overline{A+B}$.

Theorem 3.5: The set of all full k-ideals of ternary semi ring S. denoted by $\mathrm{I}(\mathrm{S})$, is a complete lattice which is also modular.

Proof. Firstly we note that $\mathrm{I}(\mathrm{S})$ is a partially ordered set with respect to usual set inclusion. Let $A, B \in I(S)$. Then by Theorem 3.1 $A \cap B \in I(S)$, and by Theorem $3.4, \overline{A+B} \in I(S)$.

Define $A \wedge B=A \bigcap B$ and $A \vee B=\overline{A+B}$.

It is clearly that $A \cap B=\inf \{A, B\}$, let $C \in I(S)$ such that $A, B \subseteq C$.

Then $A+B \subseteq C$ and $\overline{A+B} \subseteq C$. But $C=\bar{C}$.

Which implies that $\overline{A+B} \subseteq C$.

Hence $\overline{A+B}=\sup \{A, B\}$. Thus we find that $\mathrm{I}(\mathrm{S})$ is a lattice.

If $S$ be a ternary semiring, then $E^{+}(S)$ is an ideal of $S$.

Thus $E^{+}(S)$ is an ideal of $S$, which contained in every ideal in $\mathrm{I}(\mathrm{S})$.

Hence $\overline{E^{+}(S)}$ is the smallest full k-ideal in $\mathrm{I}(\mathrm{S})$, and also $S \in I(S)$.

Consequently $\mathrm{I}(\mathrm{S})$ is a complete lattice.

Finally to show that $\mathrm{I}(\mathrm{S})$ is modular.

Suppose that $A, B, C \in I(S)$ such that $A \wedge B=A \wedge C$ and $A \vee B=A \vee C$ and $B \subseteq C$.

Let $x \in C$. we have $C \subseteq \overline{A+C}=A \vee C$, so $x \in A \vee C=\overline{A+B}$.

Hence there exists $a+b \in A+B$ such that $x+a+b=a_{1}+b_{1}$ for some $a_{1} \in A, b_{1} \in B$.

Then $x+a+a^{\prime}+b=a_{1}+b_{1}+a^{\prime}$.

But $x \in C, a+a^{\prime} \in E^{+}(S) \subseteq C$

Since $C$ is full ideal and $b \in B \subseteq C$, then $a_{1}+b_{1}+a^{\prime} \in C$. But $b_{1} \in B \subseteq C$, which is k-ideal.

So $a_{1}+a^{\prime} \in C$, also $a_{1}+a^{\prime} \in A$ which implies that $a_{1}+a^{\prime} \in C \cap A=A \cap B$.

Hence $a_{1}+a^{\prime} \in B$.

So from (1), We find that $x+a+a^{\prime}+b=a_{1}+a^{\prime}+b \in B$. But $\left(a+a^{\prime}\right)+b \in B$, which is a k-ideal.

Which implies that $x \in B$.

Hence $\mathrm{B}=\mathrm{C}$.

Therefore $\mathrm{I}(\mathrm{S})$ is a modular lattice.

\section{Conclusions}

We considered the notion of $\mathrm{k}$-ideals and fully $\mathrm{k}$ ideals in ternary semirings and studied their properties and relations between them.

\section{References}

1) Vandiver HS. Note on a simple type of algebra in which the cancellation law of addition does not hold. Bulletin of the American Mathematical Society. 1934;40(12):914-921. Available from: https://dx.doi.org/10.1090/s0002-9904-1934-06003-8.

2) Reddy UN, Rajani K, Shobhalatha G. A Note on Fuzzy Bi-Ideals in Ternary Semigroups. Annals of Pure and Applied Mathematics. 2018;16(2):295-304. Available from: https://dx.doi.org/10.22457/apam.v16n2a5.

3) Lister WG. Ternary rings. Transactions of the American Mathematical Society. 1971;154:37-55. Available from: https://dx.doi.org/10.1090/s0002-99471971-0272835-6. 
4) Kar S. On quasi-ideals and bi-ideals in ternary semirings. International Journal of Mathematics and Mathematical Sciences. 2005;2005(18):3015-3023. Available from: https://dx.doi.org/10.1155/ijmms.2005.3015.

5) Rajeswari G, Amala M, Vasanthi T. Some special structures of $S^{*}$ and $A^{*}$ semirings. Indian Journal of Science;13(39):4109-4115. Available from: https://doi.org/10.17485/IJST/v13i39.1702.

6) Sen MK, Adhikari MR. Onk-ideals of semirings. International Journal of Mathematics and Mathematical Sciences. 1992;15(2):347-350. Available from: https://dx.doi.org/10.1155/s0161171292000437. 